\title{
Paediatric Pharmacotherapy and Drug Regulation - Moving Past the Therapeutic Orphan
}

\author{
Charlotte Moore-Hepburn ${ }^{1}$ and Michael Rieder ${ }^{2}$ \\ ${ }^{1}$ University of Toronto \\ ${ }^{2}$ University of Western Ontario
}

September 10, 2020

\begin{abstract}
The development of specific drug therapy for children was a paradigm changing event that transformed paediatric medical practice. However a series of tragedies involving drug treatment for children resulted in a gap developing between drug regulation and practice, with the majority of drugs used in child health care being used "off label" rendering children therapeutic orphans. Over the past two decades changes in drug regulation led by the US FDA and followed by the European Union's EMA have led to substantial changes in how new drugs with potential use in children are studied and labelled. While these changes have substantially improved labeling for new drugs, there has been much less progress with older drugs. As well while the unique challenges of conducting clinical research in children have been addressed by novel clinical trial designs, many of these innovations have not been translated into approaches accepted for the drug approval process. The regulations applying to the need for paediatric studies currently are only applicable in the United States and the European Union, and there is less impetus for paediatric labeling in other jurisdictions. This impacts on a number of issues beyond labeling, including the availability of child-friendly formulations. Finally the impact of Brexit on paediatric drug studies in the UK remains unclear and subject to on-going negotiations between the UK government and the European Union.
\end{abstract}

\section{A Brief History of Paediatric Pharmacotherapy}

While optimal treatment remains elusive for a number of serious paediatric conditions, children and youth currently benefit from a large array of safe and effective therapies for common and important diseases, ranging from meningitis to cancer. Given the current breadth and depth of available paediatric therapeutics, it may difficult to appreciate just how recent many of these transformative medical innovations have been, and how far therapy for children has advanced in a relatively short period of time.

Since the dawn of time and until the 1930's, childhood was a dangerous time. In most countries, the mortality rate for children up the age of five was often upwards of $25 \%(1,2)$. As recently as 1920, in the United Kingdom, the mortality rate for children under 5 years of age was 15\% (3), with the majority of deaths due to bacterial and viral infections. Thankfully, over the last century, this grim reality has changed, with under-4 mortality in the UK now reported at 0.77 per 1000 children. While improved public sanitation and highly effective mass vaccination programs are responsible for the majority of this decrease, disease specific therapy (1) has also contributed to significant improvements in childhood survival have driven.

This article reviews the history of paediatric pharmacotherapy, highlighting some of the unique challenges faced in the pursuit of paediatric-specific drugs, outlining the specific challenges that continue to impede drug development for infants, children and youth, and offering system-focused recommendations to forward safe and effective medications for this population in the future.

Antimicrobials and the emergence of disease-specific therapies 
Sir Alexander Fleming's discovery of the anti-microbial activity of penicillin in 1928 is often considered the first disease-specific therapy (5). In actual fact, this seminal observation did not translate into changes in care until well after the widespread use of another antimicrobial drug, sulfanilamide. The scientific community initially responded with little enthusiasm for "mould juice", and given challenges associated with producing the antimicrobial in large quantities, Fleming had initially considered penicillin to be most useful as a potential surface cleaner or as an agent to help isolate bacteria in culture (6). At the same time, in the early 1930s, Dr. Gerhard Domagk, a German physician, was exploring the use of various chemical agents to combat bacterial infections. In this work, he found that the sulphonamide groups in azo dyes offered potential as antimicrobial agents, notably the compound Prontosil. Famously, the first human to benefit from his landmark advance was his own daughter. When six year old Hildegard developed a serious Strep infection, and amputation of her arm was being considered, he treated her with Prontosil and she quickly recovered,.. In 1935, he published his work demonstrating that mice infected with Streptococci survived when administered a single dose of a synthetic azo dye (7). His work led to the introduction of Prontosil to the market shortly thereafter. Tréfouël and Tréfouël and colleagues at the Pasteur Institute subsequently determined that the active agent in Prontosil was the sulphonamide sulfanilamide (8).

The impact of the introduction of specific and effective medical therapy on health care -specifically child health care - and medical culture was substantial and permanent. As described by Lewis Thomas, the role of the physician up to the early $20^{\text {th }}$ Century had been primarily to provide supportive care to suffering patients, including providing explanations to support diagnosis and suggest outcome, with cure being reserved (in certain and limited cases) to surgeons (9). Prior to the 1940's, for most patients with infection, medical management includes watchful waiting and symptom management until the infection resolved or the patient succumbed. The establishment of effective antimicrobial therapy was a paradigm-changing event, comparable today to curing all cancer, or reversing now-irreversible neurologic injury (9). As effective antimicrobial therapy became available, the mortality rate from meningitis fell from $95 \%$ to $5 \%$. Similarly, death from pneumonia or bacterial skin infection - previously common - became rare. In parallel, the pharmaceutical industry formalized itself, and came to recognize the significant potential associated with disease-specific cures, which launched a focused search for other new molecular entities $(1,5)$. The Therapeutic Revolution that followed these fundamental discoveries first led to a wave of new treatments based on innovative small molecules and subsequently led to the revolution in biological and gene-based therapies.

For children, this focus on disease-specific therapy has been profound for some disorders. While the first and arguably largest impact was with infectious diseases, advances in the understanding of the fundamentals of paediatric drug therapy, including paediatric pharmacokinetics and the role of therapeutic drug monitoring led to substantial changes in the management of other common and serious disorders in childhood. Childhood cancer, which had a nearly universally fatal outcome less than a century ago, now boasts cure rates, for some of the commonest cancers, of 80 to $90 \%$ in large part due to highly effective therapy (10). Paediatricians and other child health care providers have proven adroit at applying medications developed for adult indications into paediatric practice, often very shortly after the drug in question entered the market.

\section{Drug Regulations and the Paediatric Promise}

The introduction of the sulphonamide antibiotics to clinical practice also indirectly had a major impact on drug regulation. While drugs have been regulated in the UK since 1540 - initially under the Apothecaries Wares, Drugs and Stuffs Act - the primary focus of concern, as was the case drug regulatory agencies in most countries up to the early $20^{\text {th }}$ century, related to identifying commercial preparations for the purpose of securingpatent and proprietary rights. Early regulatory frameworks paid little attention to the efficacy or safety of approved medications, notably with respect to use in children. A classic case study of this disregard is the approval of a product known as Dr. James Soothing Syrup Cordial, advertised as a cure for cholera infantum or infectious diarrhoea and suggested for use for colic and teething pain (11). The active ingredient in Dr. James Soothing Syrup Cordial was heroin - which the company claimed was not habit forming. Other examples include cocaine drops marketed for teething pain and marijuana cigarettes, listed on the US Pharmacopeia for headache. 
Following the introduction of the first sulphonamide preparations on the market, it became clear that the very large commercially available tablets were difficult for children to take, especially children under the age of six. To address this problem, a chemist at S.E. Massengill dissolved sulfanilamide in diethylene glycol, an effective solvent and, unfortunately, a potent nephrotoxin. With the addition of pleasantly flavored raspberry syrup, the liquid sulphonamidewas then marketed as Elixir of Sulfanilamide in September of 1937. Although the toxicity of diethylene glycol was known -and had been documented - since 1930, this had not been appreciated by the development team at Massengill. In addition, prior to commercializing the product or obtaining approval to market, no animal testing was performed and no rigorous safety appraisal was completed, as neither were required for such testing at the time- and Sadly, very shortly after Elixir of Sulfanilamide became available, a number of patients - mostly children - sustained significant renal injury and more than 100 died $(11,12)$. The public outrage following this event lead to the development and passage of the 1938 Food, Drug and Cosmetic Act, the legislation that was fundamentally responsible for the structure of the US Food and Drug Administration ("FDA") as it exists today. This legislative frameworkwhich established that Federal drug regulatory agencies have a fiduciary duty to ensure that marketed drugs are safe - has been adopted by national drug regulatory agencies worldwide.

Changes in drug policy and regulation driven by tragedies in childhood unfortunately did not end with the Elixir of Sulfonamide disaster. Despite the preliminary protections provided by the early versions of the Food, Drug and Cosmetic Act, paediatric patients remained a uniquely vulnerable population with respect to marketed drug products. As new therapies for a variety of conditions became available, paediatricians and other child health care providers rapidly adopted them into practice. This use was primarily based on literature from adult populations, as the need for paediatric testing was not required, and was rarely available. - For example, after the discovery of the antimicrobial efficacy of chloramphenicol in 1947, the drug was widely used, including for the therapy of presumptive sepsis in neonates. When used for this indication without efficacy or safety data in children - a number of babies developed cardiorespiratory collapse, termed the Chloramphenicol Grey Baby Syndrome $(13,14)$. It has subsequently been recognized that this was due to the developmental immaturity of drug metabolizing systems, specifically glucuronidation, paired with a developmentally driven reduced renal clearance of the parent drug in the neonate resulting in accumulation of toxic concentrations of chloramphenicol (15). This, in turn, produced mitochondrial dysfunction and cellular toxicity, impairing cardiac function resulting in the Chloramphenicol Grey Baby Syndrome. This tragedy was key in driving foundational research in developmental pharmacology, manifesting the truth of Abraham Jacobi's quote 1889 that "pediatrics does not deal with miniatures men and women, with reduced doses and the same class of diseases in smaller bodies, but it has its own independent range and horizon and gives as much to general medicine as it receives from it" (16).

A further tragedy - and one with equally monumental consequences for pharmacotherapy for children occurred when the German pharmaceutical company Chemie Grunenthal marketed thalidomide as a sedativehypnotic in 1957 (17). The product launch was very successful; it was marketed in a number of other European countries including the UK, and was used by more than a million women in Germany alone (18). Four years after the product launch, William McBride, an Australian obstetrician, described an association between in utero exposure to thalidomide and congenital limb malformations, more specifically phocomelia, preferential shortening of the upper limb segments which has a natural incidence of between 1 and 4 per 100,000 live births but was seen in up to $20 \%$ of women taking thalidomide over the time of embryogenesis (19). This was subsequently confirmed by Lenz, with approximately 8,000 children effected worldwide, mostly in West Germany, the UK and Japan $(1,20)$. While there were some cases in North America, there were relatively few, as thalidomide was only approved in Canada in April 1961, and subsequently withdrawn in 1962. Thalidomide was never approved in the United States due to the concerns of Dr. Francis Kelsey, a reviewer at the FDA who was troubled by reports of embryopathy from Australia and Europe. Of note, Dr. Kelsey's graduate work at the University of Chicago had included studies with Gelling exploring the toxicity of sulfanilamide.

Despite the fact that thalidomide had never been approved in the United States, knowing that thousands of children worldwide had been harmed by drug therapy during pregnancy lent urgency to critically important 
drug-related legislative changes proposed by Estes Kefauver, a Senator from Tennessee, and Oren Harris, a Representative from Arkansas. This important legislation - known as the Kefauver-Harris Amendment - was passed in October 1962, and served to strengthen the Food, Drug and Cosmetic Act in the United States. These changes made the approval of the Food and Drug Administration mandatory prior to the release of a drug to market, and granted powers to the FDA enabling them to require manufactures to demonstrate the effectiveness of their products and report serious adverse events following market authorization. These changes also transformed quality of clinical trials to be used for the purpose of drug approval, set controls for the marketing of generic products and established good manufacturing practices for industry clearly delineated the requirement that drugs must be shown to be both safe and effective in the populations for which they were indicated before they could be approved for marketing and regulated the sale and marketing of generic drugs as well as the conduct of clinical trials (21). This amendments had an immediate and profound effect on the drug approval process notably on the quality of new drug submissions, and are credited with transforming the landscape of drug development and approval.

Unfortunately, this landmark Amendment also had an equally profound and quite unintended effect on pediatric pharmacotherapy. With an aim to secure the safety of marketed pharmaceuticals, the Act stipulated that there must be substantial evidence of efficacy pursuant to the conditions of use as outlined in the product monograph. Adverse effects of the drug also needed to be studied during drug development and had to be stated in detail in the product monograph.. The unique circumstances of conducting research in children at the time created a number of pragmatic problems that made this difficult including a relative lack of clinical trial data in children, small population sizes in most centres mandating multi-centre and network studies, lack of validated end-points for clinical trials, the ethics of appropriate informed consent and cost of conducting studies. In many cases, it was determined that if the product indication did not include children there was no legislative or regulatory requirement to conduct studies in children. This frequently led to strategic decisions during drug development to not include children in clinical studies of new therapeutic agents. Therefore, as most new drugs were not studied in children prior to market authorization, to ensure compliance with the regulations, product monographs often contained exclusionary language that recommended against use of the drug in children.

This paradoxical situation where legislation intended to enhance safety testing in children produced a decrease in drug research in children, resulting in the majority of drugs used in the care of children being used "off label", that is to say without guidance from the product monograph - and often without evidence to guide clinicians as to dose or safety, a problem for children most eloquently described by Harry Shirkey in 1968 as children being "therapeutic orphans" (22).

Thus, an important amendment intended to protect all citizens from harm by strengthening the authorities of the FDA, and enforcing regulations designed to increase the safety and efficacy of prescribed medications, resulted in children being excluded from the new regulatory protections. For many years following the passage of the Kefauver-Harris Drug Control Act, there was neither incentive nor regulatory imperative to include paediatric data in new drug submissions unless the drug was specifically indicated for children.

This issue persisted for decades, despite calls by professional societies and pharmacologists for change (23-26), and despite clear and compelling evidence that there was "off label" use was becoming an unacceptable norm for paediatric practice $(23,40,41)$. Coupled with the barriers identified with respect to the conduct of clinical trials in children, there has been a persistent and incorrect belief that "children rarely require prescription medication". This, however, is simply not the case. Children are frequently prescribed medications, and for a wide range of indications (27-39). Having studied a cohort of nearly a million children in Canada for a year, researchers found that, on average, children received 4 prescriptions per year $(27,28)$. American studies have demonstrated similar numbers; in one study, $27 \%$ of all children studied had received a prescription in the past 30 days $(33,36)$. For inpatient care, a multinational study of drug utilization demonstrated that $96 \%$ of children admitted to hospital in the UK received at least one prescription medication (34).

In addition to a large number of medications being prescribed, there are large range of medication classes used (27-36). While antimicrobials were found to be the most commonly prescribed drugs for children, up to 
1200 other drugs from a wide range of classes were used (27-36). Although the use of drugs for children is to some extent variable from country to country, probably reflecting national differences in child care practices and drug availability, one trend that has emerged as well was that over time fewer antimicrobials were used and more psychoactive drugs are being prescribed $(34,36)$. To add complexity, over the past decade, the number of biological agents prescribed to children has increased almost logarithmically (37-39).

An additional consideration is that the use of pharmacotherapy for children is not uniformly distributed across the population. For many children, pharmacotherapy is indeed infrequent and largely confined to antimicrobials and respiratory medications. However for children with serious and/or chronic disease, therapy is not only frequent but also frequently involved polypharmacy $(27,28)$. As an example, it has been demonstrated that for children with epilepsy they were treated with up to seven different drugs over the first year after diagnosis (28).

\section{Progress to Date}

After years of research demonstrating the extent of this problem, coupled with advocacy by national professional societies in paediatrics and child health, there was movement in this area when the Pediatric Pharmacology Research Unit (PPRU) network was established in the United States by the Eunice Kennedy Shriver National Institute of Child Health and Human Development in 1994. This network - a group of first seven and then thirteen academic health care centre which operated from 1994 to 2010 - had the mandate to improve the paediatric labelling of both new drugs and drugs already on the market by conducting collaborative research with academia, industry and health care providers (42). Over the life span of the PPRU, a number of advances were made in understanding the impact of ontogeny on therapy for children and in the design and conduct of drug studies in infants and children.

The development of the PPRU network was accompanied by legislative change, the first being the Pediatric Labelling Rule of 1994, issued by the US FDA. This rule, which requested industry to submit paediatric data, was fairly ineffective in enhancing paediatric labelling and thus in 1997 the FDA Modernization Act (FDAMA) was passed (43). FDAMA provided incentives for companies to include paediatric data in new drug submissions by extending 6 months of patent protection for drugs when submission includes paediatric data. Failing to achieve the desired effect, in 1998, the first iteration of a "Pediatric Rule" was enacted which mandated paediatric studies for not only new drugs but also for any label changes on already approved and marketed drugs. This rule, however, was challenged in Federal court and was ultimately struck down in 2002, citing Agency overreach.

Despite this early setback, the United State Congress persisted, and the Best Pharmaceuticals for Children Act (BPCA) passed in 2002 while the Pediatric Research Equity Act (PREA) passed a year later in 2003. Together, these two pieces of legislation imposed a regulatory requirement for pharmaceutical companies to evaluate drugs in paediatric populations if they were likely to be used in children, irrespective of the indication for which approval was sought (43).

The provision of paediatric exclusivity was successful in having paediatric-specific data on the project monograph when an FDA request for paediatric data was issued in $92 \%$ of all requests for which exclusivity was granted from 1998 to 2012 (44). This provision allows six additional months of patent exclusivity to manufacturers who conduct paediatric drug studies and have a Pediatric Study Plan (PSP). However, of 401 requests made by the FDA exclusivity was only granted in 189 cases, i.e. the majority of requests did not result in new paediatric data or indications (44). This was influenced by both therapeutic area and year of exclusivity, with requests made in the areas of gastroenterology and pain and requests made in the early years of the program being more likely to result in additional paediatric data or indications (44).

Similar regulatory progress has been made internationally. In Europe, the European Medicines Agency (EMA), after studying developments in the United States, enacted the Paediatric Regulation in 2007 with the goal of enhancing the development, availability and safety of medications for children in the European Union (45). An important aspect of the Regulation was the requirement for a Paediatric Investigation Plan (PIP) for all new drug submissions except when a waiver or deferral is granted, as in the case of a drug 
that will not in any reasonable likelihood used for children. The Regulation also established a Paediatric Committee to determine which studies must be carried out as part of the PIP. The FDA requires a Pediatric Study Plan and also has a Pediatric Expert Committee.

A ten year analysis of the implementation of the Paediatric Regulation demonstrated that there had been a substantial increase in the number of medications with paediatric data and/or indications, notably in the areas of rheumatology and anti-infective agents. However, in diseases largely confined to children alone or where there were substantial differences in disease course between adults and children there was much less progress. As a consequence, the EMA and the Paediatric Committee created an action plan to enhance implementation of the Regulation. The action plan includes identifying paediatric medical needs, strengthening cooperation between decision makers, ensuring the timely completion of PIPs, improving how PIPs are handled and increasing transparency around paediatric medicines.

While both the US FDA and the European Union's EMA had common goals to enhance the development of more safe and effective therapeutics for children they have pursued slightly different approaches (Table 1) (45). As an example, the FDA had two separate sets of legislation - BCPA and PREA - to drive drug development for children with a third - Research to Accelerate Cures and Equity for Children Act (the RACE Act) being introduced in 2017 while the EU has a single piece of legislation, reducing the number of requirements and legal frameworks to be negotiated. This does not, however, necessarily mean that it is easier to navigate the EMA's paediatric requirements as opposed to the FDA. While there are other differences overall this legislative approach in both the US and the European Union not only is the goal the same but so are the scientific elements driving paediatric investigation, including but not restricted to descriptions of products and diseases, development plans for age-appropriate formulations and timing of studies (45).

The developments in the US and European Union to move paediatric drug development forward have stimulated changes in the approach to paediatric drug approval and regulation in a number of countries although the US and the European Union remain the only two large jurisdictions to have comprehensive paediatric drug development strategies supported by legislation although some smaller jurisdictions such as Switzerland that require paediatric data, often via the approval of an approved PIP or PSP (26, 46-48).

\section{Future Challenges}

As we look to continue to strengthen both clinical and regulatory protections for children, a number of significant challenges require the attention of paediatricians, pharmacologists, pharmacists and policy makers.

Off Label Use. The regulatory environment for new drug approvals in the United States and the European Union has resulted in an increase in paediatric labelling for new therapeutic entities and a parallel decrease in off-label use (49). This is an important issue. While off-label drug use does occur in adults, this is typically on the basis of new evidence that demonstrates safety and efficacy of drugs for new indications. In contrast, in children off-label drug use has often been on the basis of extrapolation in the absence of firm evidence, and on many occasions has been associated with adverse outcomes. Off label drug use has been identified as a separate and distinct risk factor for adverse drug reactions in children $(49,50)$.

However, the decrease in off-label use in Europe has been marginal despite these changes $(64 \%$ post legislative changes versus $58 \%$ prior the changes) (50). This is likely due to the fact that the existing regulatory mechanisms address only novel products, and the majority of drug use in children is concentrated in older, often off-patent medications. The best approach to enhancing paediatric labelling and reducing off-label use, notably for older medications, remains unclear.

Child Friendly Formulations. The issue of child-friendly formulations remains an important issue and one that, in pragmatic terms, often "orphans" children from safe and effective therapies (1). Many children under the age of eight and essentially all children under the age of four have difficulty in swallowing conventional tablets. (Interestingly, up to $10 \%$ of adults and a higher number of senior citizens report difficulty in swallowing conventional tablets and capsules). Some medications - notably antibiotics - are available in liquid form but for many other medications pharmacists or parents must crush and dissolve 
or mix medications with flavour masking agents (51). Creating a dosage form in this manner - a practice known as compounding - is a skill that is less and less frequently taught in pharmacy faculties and is often quite variable in approach and technique. Compounding introduces a new series of risks including dose and drug errors. Importantly, compounding also circumvents the carefully designed regulations for Good Manufacturing Practice established to provide safe dosage forms for adults.

There have been a number of innovative approaches to enhance oral drug delivery for children, and while many such as mini-tablets, gel technology and 3D printing onto orodispensible films offer great promise, their uptake has been less rapid and certainly less generalized than desired (52). In the area of child-friendly formulations, European research groups have been among the world leaders (53). However, the dissemination of these formulations beyond Europe and the United States is much at best spotty and certainly less than optimal (47). Work in a number of centres and by a number of regulators is exploring the safety and optimal nature of excipients in paediatric formulations.. Given that children will likely always represent a small market for pharmaceuticals compared to adults, it is likely that this work would benefit substantially form incentives to counterbalance the impact of market forces on the impetus for formulation development.

Design of Clinical Trials in Children. A further regulatory challenge lies in the area of clinical trial design. While regulatory approval has relied heavily on conventional randomized double-blind controlled trials - the "gold standard" for drug approval - this has been problematic for drugs for children, related to issues such as small sample size, selection of appropriate and clinically meaningful outcomes, appropriate instruments for evaluation and suitable safety monitoring (26). To address these issues and to meet the challenges of the new revolution in therapeutics, investigators have developed a number of novel clinical trial designs tailored to the unique problems in paediatric drug research (55-58). Examples of these include adaptive design, in which trial design is changed over the course of a trial based on interim analysis; this is a different approach than the conventional randomized double blind trial and requires meticulous pre-study planning (55). A challenge for drug regulatory agencies is how to best accept the results of these studies for drug approval and to inform product monographs.

Regulatory Harmonization. An additional challenge for drug development in general but for paediatrics in particular is harmonization of requirements and regulations across multiple jurisdictions. The International Council for Harmonization of Technical Requirements for Pharmaceuticals for Human Use (ICH) is a Swissbased group that for the past twenty-five years has been working to harmonize the regulatory requirements of multiple jurisdictions. Over the past decade, many jurisdictions have modified their requirements for new drug submissions to better align across international boundaries, and although there has been some success in this area much work remains to be done (54). The frequent and increasing dialogue between the FDA and EMA as to how to encourage drug studies in children is an encouraging development, notably given that regulators for other jurisdictions are involved in these discussions as observers.

Brexit. In addition to global issues there are interesting challenges in how Brexit will impact on paediatric drug development in the UK - which has a number of talented paediatric clinical pharmacologists and some of the best academic child health care centres in Europe - has yet to be determined (59). The UK academic and industrial community and EMA have benefited greatly from close interaction and it is hoped that whatever agreements are negotiated as the UK leaves the European Union can preserve these benefits.

Talent Pool and Pipeline. A challenge and an opportunity is the talent pool of researchers with interest and expertise in conducting drug research in children. The number of investigators is limited and primarily located in developing countries (60). In part this is due to the perception that immediate clinical needs in developing nations may be seen to be a higher priority than the development of drug researchers and is in part due to the fact that promising young investigators who train in the developing world are often attracted to the deeper resources available for research in the developed world. However, there is a cadre of young pharmacologists with interest in moving drug research in children forward and in working together across countries and across regions in creating the critical mass needed to surmount the challenges detailed above.

\section{Summary and Direction Forward}


The unintended consequences of well intentioned actions produced a situation in which children were therapeutic orphans, often denied the benefits of research and evidence in guiding therapy. While there has been considerable progress over the past two decades in addressing this issue, much remains to be done, notably as the next generation of therapeutics in the form of biologics, cell therapy and genetic engineering evolve. Fortunately there is will and energy, notably among the next generation of child health care providers and researchers, to address and surmount these challenges in pursuit of the common goal of effective and safe therapy for children everywhere.

\section{References}

1. Rieder MJ. If children ruled the pharmaceutical industry: The need for pediatric formulations. Drug News Perspect 2010; 23: 458-64.

2. Bideau A. Desjardins B, Perez Brignoli H (eds). Infant and Child Mortality in the Past. Claredon Press, Oxford, 1997.

3. Kermack W, McKendrick AG, McKinley PL. Death Rates in Great Britain and Sweden: Some general regularities and their significance.Lancet 1934; 31: 698-703.

4. UN Inter-agency Group for Child Mortality Estimation, https://childmortality.org

5. Fleming A. On the antibacterial action of cultures of a penicillium with special reference to their use in the isolation of B. influenzae. Br J Exper Path 1929; 10: 226-36.

6. Weinshilboum RM. The therapeutic revolution. Clin Pharmacol Ther 1987; 42: 481-5.

7. Domagk G. Eine neue klasse von desinfektionshmittelm. Deutsch Med Wochenschr 1935; 61: 829-32.

8. Trefouel J, Trefouel J, Nitti F, Bovet D. Activite du p-aminophenyl-sulfamide sur les infections stretococciques experimentales de la souris et du lapin. Cr Scanc Soc Biol1935; 120: 756-8.

9. Thomas L. The youngest science: Notes of a medicine-watcher. Viking Press. New York, 1983.

10. O'Leary M, Krailo M, Reaman GH. Progress in childhood cancer: 50 years of research collaboration, a report from the Children's Oncology Group. Semin Oncol 2008; 35: 484-93.

11. Connolly CA. Drug Therapy: From 'Baby Killers' to Baby Savers, 1906-1933." In: Children and Drug Safety: Balancing Risk and Protection in Twentieth-Century America, by Cynthia A Connolly, Rutgers University Press, New Brunswick, Camden, Newark, New Jersey; London, 2018, pp. 1-15.

12. Wax PM. Elixirs, diluents and the passage of the 1938 Federal Food, Drug and Cosmetic Act. Ann Intern Med 1995; 122: 456-61.

13. Sutherland JM. Fatal cardiovascular collapse of infants receiving large amounts of chloramphenicol. Am J Dis Child 1959; 97: 761-7.

14. Mulhall A, de Louvois J, Hurley R. Chloramphenicol toxicity in neonates Its incidence and prevention. Br Med J 1983; 287: 1424-6.

15. Kearns GL, Abdel-Rahman SM, Alander SW et al. Developmental pharmacology - drug disposition, action and therapy in infants and children. N Engl J Med 2003; 349: 1157-67.

16. Burke EC. Abraham Jacobi, MD: The man and his legacy. Pediatr1998; 101: 309-12.

17. Burgio GR. The thalidomide disaster revisited. Eur J Pediatr 1981; 136: 229-30.

18. Vargesson N. Thalidomide induced teratogenesis: History and mechanisms. Birth Defects Res C Embryo Today 2015; 105: 140-56.

19. McBride WG. Thalidomide and congenital malformations.. Lancet1961; 2: 1358.

20. Lenz W. Kindliche missbildungen nach medikameteinnahme wahrend der graviditat? Dtsch Med Wochensche 1961; 86: 2555-6.

21. Kauffman RE. Status of drug approval processes and regulation of medications for children. Curr Opin Pediatr 1995; 7: 195-8.

22. Shirkey HC. Therapeutic orphans J Pediatr 1968; 72: 119-20.

23. Kauffman RE. Essential drug for infants and children: North American perspective. Pediatr 1999; 104 (S3):603-5.

24. Committee on Drugs, American Academy of Pediatrics. Use of drugs not described in the package insert (off-label uses). Pediatr 2002; 110: 181-3.

25. Pandolfini C, Bonatii M. A literature review on off-label drug use in children. Eur J Pediatr 2005; 
164: $522-8$.

26. MacLeod SM. Current environment for drug development, regulation and use. In: Improving Medicines for Children in Canada. Council of Canadian Academies, Ottawa, Ontario, 2014.

27. Rieder MJ, Matsui D, MacLeod S: Myths and challenges - Drug utilization by Canadian children. Paed Child Health 2003; 8: 7A-8A

28. Khaled LA, Ahmed F, Brogan T et al. Prescription drug use by one million Canadian children. Paed Child Health 2003; 8 (Supple A), 1-51.

29. Lasky T. Estimates of pediatric medication use in the United States: current abilities and limitations. Clin Ther 2009;31:436-45.

30. Turner MA, Lewis S, Hawcutt DB, Field D. Prioritising neonatal medicines research: UK Medicines for Children Research Network scoping survey. BMC Pediatr 2009 Aug 12;9:50.

31. Vernacchio L, Kelly JP, Kaufman DW, Mitchell AA. Medication use among children <12 years of age in the United States: results from the Slone Survey. Pediatr 2009;124:446-54.

32. Clavenna A, Bonati M. Drug prescriptions to outpatient children: a review of the literature. Eur $J$ Clin Pharmacol 2009;65:749-55.

33. Chai G, Governale L, McMahon AW, Trinidad AW Trinidad JP, Staffa J, Murphy D. Trends of outpatient prescription drug use in US children, 2002-2010. Pediatr 2012 ;130:23-31.

34. Rashed AN, Wong ICK, Wilton L, Tomlin S, Neubert A. Drug utilization patterns in children admitted to a paediatric general medical ward in five countries. Drugs Real World Outcomes 2015; 2: 397-410.

35. Rosli R, Dali AF, Abd Aziz N, Abdullah AH, Ming LC, Manan MM. Drug utilization on neonatal Wards: A systematic review of observational Studies. Front Pharmacol 2017; 8:27.

36. Martin CB, Hales CM, Gu Q, Ogden CL. Prescription drug use in the United States, 2015-2016. NCHS Data Brief 2019; 334.

37. Swart J, Giancane G, Horneff G et al. Pharmacovigilance in juvenile idiopathic arthritis patients treated with biologic or synthetic drugs: Combined data of more than 15,000 patients from Pharmachild and national registries. Arthritis Res Ther 2018; 20: 285.

38. Griesenauer RH, Kinch MS. 2016 in review: FDA approvals of new molecular entitites. Drug Discov Today 2017; Jul 4. pii: S1359-6446(17)30129-0.

39. Mahmood I. Pharmacokinetic considerations in designing pediatric studies of proteins, antibiotics and plasma-derived products. Am J Ther 2016;23:e1043-56.

40. Wilson JT. Update on the therapeutic orphan. Pediatr 1999; 104: 585-9.

41. Aronson JK, Ferner RE. Unlicensed and off-label uses of medicine: Definitions and clarification of terminology. Br J Clin Pharmacol 2017; 83: 2615-25.

42. Cohen SN. The pediatric pharmacology research unit (PPRU) network and its role in meeting pediatric labeling needs. Pediatr 1999; 104: 644-5.

43. Institute of Medicine (US) Forum on Drug Discovery, Development, and Translation. Addressing the Barriers to Pediatric Drug Development: Workshop Summary. Washington (DC): National Academies Press (US); 2008. 2, Regulatory Framework.

44. Wharton GT, Murphy MD, Avant D et al. Impact of pediatric exclusivity on drug labelling and demonstrations of efficacy.Pediatr 2014; 134: e512-8.

45. Penkov D, Tomasi P, Eichler I et al. Pediatric medicine development: An overview and comparison of regulatory processes in the European Union and the United States. Ther Inno Regul Sci 2017; 51: 360-71.

46. Thomsen MDT. Global pediatric drug development. Curr Ther Res2019; 90: 135-42.

47. Moore Hepburn C, Gilpin A, Autmizguine J et al. Improving paediatric medications: A prescription for Canadian chidren and youth.Paeds Child Health 2019; 24: 333-5.

48. Saitou H, Nakatani D, Myoui A, Kubota, Ozono K. Pediatric drug development in Japan: Current issues and perspectives. Clin Pediatr Endocrinol 2020; 29: 1-7.

49. Committee on Drugs, American Academy of Pediatrics. Off-label use of drugs in children. Pediatr 2014, 133 563-567.

50. Corny J, Lebel D, Bailey B, Bussieres JF. Unlicensed and off-label drug use in children before and 
after pediatric government incentives.J Pediatr Pharmacol Ther 2015; 20: 316-28.

51. Rieder MJ. Size and Taste Matters: Recent Progress in the Development of Age-Appropriate Medicines for Children. Pharmaceut Med2018; 32:21-30.

52. Gerrard SE, Walsh J, Bowers N, Salunka S, Hershenson S. Innovations in pediatric drug formulations and administration technologies for low resource settings. Pharmaceutics 2019; 11: doi:10.3390/pharmaceutics 11100518.

53. Thabet Y, Klingmann V, Breitkreutz J. Drug formulation: Standards and novel strategies for drug administration in pediatics. J Clin Pharmacol 2018;58 Suppl 10:S26-S35.

54. Teixeira T, Kweder SL, Saint-Raymond A. Are the European Medicine Agency, US Food and Drug Administration, and other international regulators talking to each other? Clin Pharmacol Ther 2020: 107: $507-13$.

55. Rieder M, Hawcutt D. Design and conduct of early phase drug studies in children. Br J Clin Pharmacol 2016;82:1308-1314. PMID: 27353241

56. Duffett M, Swinton M, Brouwers M, Meade M, Cook DJ. Advancing randomized controlled trials in pediatric critical care: The perspectives of trialists. Pediatr Crit Care Med2018;19:e595-e602.

57. Cohen JW, Akshintala S, Kane E, et al. A systematic review of pediatric Phase I trails in oncology: Toxicity and outcomes in the era of targeted therapies. Oncologist 2020 Jan 14 . doi: 10.1634/theoncologist.2019-0615.

58. Collingnon O, Gartner C, Haidich A-B et al. Current statistical considerations and regulatory perspectives on the planning of confimatory basket, umbrella and platform trials. Clin Pharmacol Ther 2020; 107: 1059-67.

59. Breckenridge A, Feldschreiber P. Impact of Brexit on UK and EU Drug Regulation and Patient Access. Clin Pharmacol Ther 2019; 105: 923-5.

60. MacLeod SM, Greff M, Knoppert DC, Ito S, Rieder MJ. An international asset map of clinicians, educators and researchers pursing better medicine use in children: Initial findings. Clin Pharmacol Ther 2017; 101: 274-80.

\section{Hosted file}

Table 1 Paeds Pharmacotherapy - Regs.docx available at https://authorea.com/users/ 355684/articles/478822-paediatric-pharmacotherapy-and-drug-regulation-moving-past-thetherapeutic-orphan 